sponses under both natural and artificially produced habitats receiving initial attention. The results so far are distinctly favourable to the use of the plant as habitat measure ; a short and preliminary account is to be published in the near future. For the forester, phytometric cultures, either free or under control, seem likely to prove valuable in returning information regarding the habitat as it impresses itself upon the plant, in throwing light on practices and processes either detrimental or conducive to the production of the optium yield from particular localities, and in indicating the species best suited to the areas to be planted.

By many it is realised that the most elaborate measurement of the physical factors of the habitat by instrumental methods usually does not even approximately inform the investigator as to the nature of that habitat as it influences the living plant itself. The plant is the truest measure of the effects of the complex produced by the integration of the several habitat factors. Provided that points of such importance as the careful selection of suitable types of plants, the employment of large numbers of individuals so that abnormal ones can be eliminated, and the study of the several responses of the plant in conjunction with physical apparatus, receive careful attention in the first instance, it does not seem too optimistic to believe that, with increased knowledge of the behaviour of selected species, the phytometer of itself will give intelligible quantitative information as to the nature of the habitat.

The value of the phytometer for analyses of habitat and vegetation processes alike is likely to be as great as the study of this grand subject is alluring.

Forest Research Station,

JohN Phillips.

Deepwalls, near Knysna, South Africa, November $2 I$.

\section{The Principal Series of the Copper Arc Spectrum.}

THE second pair of the principal series of the arc spectrum of copper has been calculated by Randall (Astro. Jour., 34, I, I9II) as 2025.73 and 2024.42 (Rowland scale). Kayser and Runge (Kayser, " Handbuch der Spectroscopie," vol. 5) observed only one line at 2025.08 (I. A.), while two lines in this region have been photographed by Rubies (An. Soc. Esp. $Y$ Quim, I5, 432, I9I7), and their wave-lengths given in Fowler's Report on Series in Line Spectra as 2025. I and 2024.II (presumably on the Rowland scale). Shenstone (Phil. Mag., 49, 95I, 1925) in his low voltage arc work found only one line and gives its wave-length as $2024 \cdot 33$.

Recently in this laboratory photographs of the arc spectrum of copper under varying conditions have been taken on several large quartz spectrographs. The dispersion of these instruments at $\lambda 2025$ is approximately $0.8 \mathrm{~mm}$. per Angström unit. The exposures taken varied from $I_{5}$ to 30 minutes. The current strength of the arc was 3 amperes on a 220 volts circuit.

When the middle of the arc was projected on the slit of the instrument, the 2024 line alone was recorded. When the whole image of the arc was projected, both lines were recorded, but the 2025 line was shown at the poles only. In all photographs the 2024 line was shown reversed. A plate of the spark spectrum showed only 2025. This agrees with Hasbach's spark work.

Independent calculations were made from photographs taken on three separate instruments. Interpolations were taken from a Hartmann formula based on the lines 2043.7 I, 2035.74 and I999.95 (Hasbach, Kayser and Konen's "Handbuch der Spectroscopie," vol. 7). The values of the 2025 line agreed to 0.01 and those of the 2024 line to 0.02 . Schumann plates were used. The following table contains our measurements, together with other known values. All wavelengths are reduced to I. A.

TABLE I.

$2024 \cdot 33 \quad 2025 \cdot 67 \quad 32 \cdot 7$

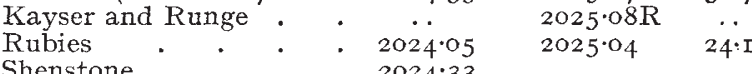

Shenstone . * $\quad 2024.33 \quad 025.36 \quad$..

Simeon and Dreblow $\quad \cdot \quad \begin{array}{ccc}2024 \cdot \mathrm{IgR} & 2025 \cdot 36 & 2025 \cdot 34\end{array}$

Our observed value of $\Delta \nu$, which is not expected to be in error by more than $\pm \mathrm{r} \cdot 0$, is notably less than Randall's calculated value.

From the results given above it is concluded that the 2024 line is a true arc line, being reversible and present in the centre, that the 2025 line is a spark line, and that therefore they do not constitute the second pair of the principal series.

F. SimEon.

E. S. DREBLow.

Research Laboratory, Adam Hilger, Ltd. 24 Rochester Place, Camden Road, London, N.W.I, November I9.

The Action of Silica on Electrolytes.

Owing to absence from the laboratory during the summer, I have only recently been able to make experiments on the effect of silica on acids, using silica prepared as described by Prof. Mukerjee in his letter to NATURE of August 29. The silicon tetrachloride was added to water in a silica dish and the mixture dialysed until the specific resistance rose to I20,000 ohms. It was then dried at the air temperature without the use of any desiccating agent. I have been unable, however, to detect in this product the slightest adsorbing power for hydrochloric acid. In his letter of April 4, Prof. Mukerjee gives figures from which $\mathrm{I}$ infer that he found that silica could take up more than I per cent. by weight of hydrochloric acid. I used one gram of silica and Ioo c.c. of $\mathrm{N} / 500$ acid and should easily have detected the adsorption of less than 0.0004 gram hydrochloric acid (i.e. 0.04 per cent. by weight) by the conductance and $p H$ measurements used. But not the slightest sign of removal of hydrochloric acid from solution was obtained.

I do not know whether Prof. Mukerjee has modified his views since April 4, as in his last letter the only experimental evidence suggested is that it is easier to remove traces of hydrochloric acid from silica by means of potassium nitrate solution than water. This is a very different matter from that which has been under discussion, and I will not take up space with a consideration of the various possible explanations. I am only concerned with the direct question as to whether or not pure silica can remove acids from solution, and I have been unable to obtain any evidence that it is able to do so.

One point has to be borne in mind when making these experiments: silica, prepared as above and partially dehydrated, retains much moisture (mine contained 20 per cent. after air drying for a week), which may dilute quite noticeably the solution to which it is added, and I have always corrected for this. The correction may be important in some cases, although in these experiments the quantities of reagents have been chosen so that it is very small.

A. F. JosEPH.

Wellcome Tropical Research Laboratories, Khartoum, November Io

NO. 293I, VOL. II 7 ] 